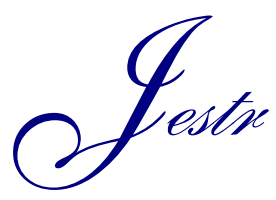

Journal of Engineering Science and Technology Review 4 (3) (2011) 271 - 276

Special Issue on Econophysics

JOURNAL OF

Engineering Science and

Technology Review

Research Article

www.jestr.org

\title{
Balanced Scorecard as a strange attractor tool contributing to the improvement of transformation process and ultimately to the competitive advantage of an Organization
}

\author{
P. Curtis ${ }^{1}$, M. Hanias ${ }^{2}$ and P. Antoniades ${ }^{3}$ \\ ${ }^{1}$ TEI of Chalkis Accounting Dep. 34400, Psachna Evias, Greece \\ ${ }^{2}$ Dep. of Electronics, Computers, Telecommunications, and Control, Faculty of Physics, University of Athens, Panepistimiopolis, Athens \\ 15784, Greece \\ ${ }^{3}$ Dep. of Electrical Engineering, Kavala Institute of Technology, St. Loukas 65404 Kavala, Greece.
}

\begin{abstract}
Organizations today represent dynamic complex systems and their behaviour cannot be examined effectively through the reductionist view of it. Competitive advantage is the ability to exploit opportunities that occur inside and outside the organisation, and adapt to meet these opportunities. It is the alignment of external and internal environments of an organization, which create and sustain competitive advantage. The use of the Balanced Scorecard (BSC) as a performance measure and a strategic control tool, contributes to organization's survival and success. The adoption of the BSC contributes to certain type of behaviour, which is elicited through the use of positive feedback. Acceptable actions are reinforced through the appropriate culture (norms and values ) and rewards linked to performance. As the pace of change is increasing, there is a constant demand for creativity and innovation. Agile and flexible BSC serves as strange attractors in the system's agents behaviour, operating in a turbulent dynamic environment. Feedbacks and information distributed throughout the system, upgrading knowledge and ultimately improving performance through learning. Organizations that are able to learn from their environment and change their internal structure survive and prosper through transformation, which is substantially influenced by strange attractors. Chaos theory can help us to understand and improve the transformation process and through the use of the BSC.
\end{abstract}

Keywords: Systems, Competitive advantage, Chaos Theory, Strange attractors, Balanced Scorecard

\section{Introduction}

Systems, chaos theory and Dynamic capabilities of Organizations

Organizations can not be understood properly, by examining separately all the elements that comprise them as reductionism suggests, since the whole is not just the sum of all the factors involved. The systems perspective of organizations on the other hand, views them as constantly interacting with their environments. The organizational environments are comprised of a nest of relationships between agents and other factors that can affect an organization and may be beyond the control of it, especially if we consider the external one. The behaviour of each element of the organization has an effect on the others and finally on the entire system. The behaviour of the elements and their effects on the whole are interdependent.

The external environment is generally beyond the control of any organization. It consists of those factors and events

*E-mail address: pcurtis@teihal.gr

ISSN: 1791-2377 @ 2011 Kavala Institute of Technology. All rights reserved. that can influence its activities. Open systems approach views the organizations' interactions with their external environment as vital for organizational survival and success. In open systems, any change in anyone element of the system, causes changes in other elements of it. An alignment between the organization and its external environment is necessary for survival and further progress. The competitive advantage of an organization and its prosperity presupposes the alignment of external and internal environments, and consequently a transformation process is necessary when the changes in the environments are constant and profound.

Chaos refers to the dynamics of an open system that at first glance has no order, but in which there is a hidden one. These systems are deterministic (with no random elements), aperiodic, not predictable in the long run and extremely sensitive to initial conditions. In these systems, small initial changes can cause complex changes in the overall system. Chaos theory applies to complex nonlinear dynamics systems, in which outputs (effects) are not strictly proportional to inputs.

Chaos and complexity theories provide the background to explore turbulent environments and view change as a 
continuous process that has to be managed successfully in order for the organization to attain, upgrade and sustain competitive advantage, that leads finally to superior performance. Organizations are complex systems. In that kind of environment the demand for an equally changing, agile and adjusting organizational structure is necessary for success and prosperity.

The resource based view (RBV) of an organization supports the idea that the source of competitive advantage and of the value creation for the organization rests predominately on the internal environment of it. Resources, capabilities and competencies are the primary sources of the value creation process and of the business prosperity. Dynamic capabilities represent a fairly recent development of the resource based view of strategy. Teece [1] define dynamic capabilities as an organization's ability to "integrate, build, and reconfigure internal and external competences to address rapidly changing environments". Dynamic Capability is an organization's collective ability to create sustainable competitive advantage by maintaining, developing further and renewing its capabilities through continuous learning by exploiting individual, organizational and environmental elements such as resources, skills, systems, structure and culture.

Organizations, based on the above, are not homogeneous with respect to their resources and capabilities endowments, since dynamic capabilities is an emergent feature and it is organization specific [1]. Dynamic capabilities do offer a competitive advantage for an organization, since they are valuable, rare, not easily substitutable and replicable and they are usually built up over time with learning mechanisms, that are specific to an organization.

Performance differences in organizations still arise due to the costs and timing of introduction of dynamic capability, but also to the effectiveness of the mechanism of appropriation of the value added of the end product. Learning processes help shape strategies toward a competitive advantage and value creation.

\section{Systems dynamics and the transformation process}

The system dynamics are studied through the use of chaos theory, which explains that a system could exhibit behaviour that would be unpredictable A change in a nonlinear system's inputs, will not lead to a directly proportional change in the outputs of the system. Dynamic systems produce outcomes that appear to bear no relationship to the changes in system input. Non-linear relationship can help to control a system by keeping it within the boundaries, through feedback mechanisms. So, small changes are reinforced and amplified into large ones, when the feedback is positive. Small actions will lead finally to major outcome that cannot be predicted.

According to [2] for a system to be healthy, it must coevolve with its environment. It must evolve in response to changes in its environment, while the later is affected also. Co-evolution is caused by disequilibrium and positive feedback (negative feedback on the other hand aims at maintaining the system the way it is). Equilibrium is defined as "a condition in which all acting influences are cancelled by others, resulting in a stable, balanced, or unchanging system". Open systems to stay viable, should maintain a state of non-equilibrium. They participate in an open exchange with their world, using inputs from it for their own growth and delivering to it the analogous output.
A perturbance, is any change in a system's environment that causes disequilibrium in a system The later creates a state in which the system is ripe for transformation and reorganization on a higher level of complexity. Transformation occurs through a process called emergence and it is strongly influenced by "strange attractors," which are a kind of fractal. Fractals are patterns that recur at all levels of a system, called self-similarity. In organizational context they can be considered "core ideas" and values or beliefs that guide or characterize the design of the system. Self-organizing systems are adaptive, they evolve themselves; they are particularly active and dynamic. Towards that end they need two major characteristics: openness and self-reference [2].

When a system is at equilibrium, its nonlinear creative potential does not reveal itself altering inputs and processes is required for the system to achieve target output through feed backing with necessary information [3]. Bifurcation points are the abrupt changes in a system's structure. It leads the system either to disorganize or "reorganize into a new form capable of attenuating or even resolving the tension". Changes within a nonlinear system are governed by an attractor. As nonlinear systems go through far-fromequilibrium conditions, the various attractor potentials are put into action. It continually bifurcates until it reaches the strange attractor state, which has boundaries and shape. The specific pattern is unpredictable, depending on the unique experience of the system and it is constraint by the culture system in it.

Strange attractors are examples of fractals that refer to patterns repeated continually.

critical point of instability far from equilibrium take place and highly organized patterns emerge that characterize the complex adaptive systems, main pillar of which is chaos theory.

The so called bounded instability ( and the associated creativity), comes about due to the coexistence of both randomness (at the individual level) and order (at the aggregate level). Bounded instability results from agent's individual movements which are random, but never exceed certain boundaries specified by the attractors. The selforganisation process, which follows destabilization, invokes a transition to a new state of bounded instability. Failure of the existing system, brings about reorganization that it is more capable to deal with its new environment. According to [4], knowledge of the bounded instability and self-organisation principles suggests that management should focus on aggregate outcome, but should not try to control individual workers' behavior. Self-organisation will result in a system better suited to the environment, with its own implicit order, caused by reorganisation around the strange attractor. self-organisation ( bifurcation) takes place as sets of strange attractors are competing within a single system [4]. Self-organization is the ability of the system, which operates far from equilibrium to defy disorder (entropy) spontaneously (without outside guided) intervention, that allows it to become more fit given the prevailing environment. This of course is possible through the flow of energy, material and information through its boundaries (since it is an open system) eschewing entropy, which is inevitable outcome based on the second law of thermodynamics.

Chaos theory and the sciences of complexity can help us to understand and possibly improve the transformation process. They can help us to understand the present system and when it is ready for transformation, and the system 
dynamics that are likely to influence changes we try to make, the effects of those changes and how it is likely to respond to changes that we try to make. Readiness for transformation is influenced by perturbations from outside the system that produce a state of disequilibrium in the system [2]. That disequilibrium may be caused by either of two kinds of changes in the external environment ones that create problems for the system and others that present opportunities to the system. At the same time there must also be sufficient enablers of transformation, which are created by factors inside the system, such as participatorydemocratic leadership, appropriate culture and beliefs.

The strange attractor denotes the path the system takes to explore its fitness landscape, continually seeking ways to higher peaks on the landscape. At each transitory or bifurcation point, the system is destabilised and switches to a new attractor. The most powerful strange attractors are core ideas and beliefs, which represent the glue substance keeping the organization united. Appropriate values and beliefs are consider to be the cornerstone of the open organisations

\section{Strategy and Controls}

Measuring the effectiveness and efficiency of strategy, is an important factor determining the success of an organization. Management controls tools in general provide a framework to monitor, measure, and evaluate accurately whether or not the organization has achieved its goals and strategic objectives. Financial and output controls must be backed up with behavior controls and organizational culture to ensure that the firm is achieving its goals in the most efficient way possible. In general, these controls should reinforce one another, and care must be taken to ensure that they do not result in unforeseen consequences, such as competition among functions, divisions, and individuals. changing organizational culture is a difficult and takes considerable time and effort. This difficulty arises because culture is the product of the complex interaction of many factors, such as top management, organizational structure, and the organization's reward and incentive systems [5]. Acceptable actions are reinforced through the appropriate culture ( norms and values ) and a reward system linked to performance

The Performance Measurement according to [6] is concerned with:

- Measuring the efficiency and effectiveness of actions;

- Aggregating and standardising information;

- Setting appropriate targets

A Performance Measurement System according to [7] should perform the following functions:

- Tracks the performance of an organisation,

- Supports company internal and external communication regarding performance,

- Helps managers by supporting both tactical and strategic decision-making,

- Captures knowledge in a company, and facilitates organisational learning

The important issues in designing an effective control system, concern the realization that the:

Organizational structure does not operate effectively unless the appropriate control and incentive systems are in place to shape and motivate employees' behaviour.
Strategic control is the process of setting targets and monitoring, evaluating, and rewarding organizational performance.

Control takes place at all levels in the organization-corporate, divisional, functional, and individual. Effective control systems are flexible, accurate, and able to provide quick feedback to strategic planners. Control systems range from those directed at measuring outputs to those measuring behaviours or actions.

Output controls establish goals for divisions, functions, and individuals. They can be used only when outputs can be objectively measured and are often linked to a management by objectives system.

Behaviour controls are achieved through budgets, standardization, and rules and procedures.

Organizational culture is the collection of norms and values that govern the way in which people act and behave inside the organization. An organization's culture is the product of a founder's or top-management team's values and attitudes, of the way managers choose to design the organization's structure, and of the strategic reward systems managers use to shape and motivate employees' behaviour.

An organization's reward systems constitute the final form of control. A company designs its reward systems to provide employees with the incentives to make its structure work effectively and to align their interests with organizational goals and objectives.

Organizations use all these forms of control simultaneously. Management must select and combine those that are consistent with each other and with the strategy and structure of the organization [5].

According to [8] two types of control strategies exist. The first one, is achieved through the performance evaluation process which entails monitoring performance and rewarding the acceptable one. The alternative one, emphasizes the minimization of preferences and goals divergence and it is achieved through selection, training and socialization of the members of the organization (social controls). The choice of the appropriate system of controls contributes considerably to the effectiveness. The behavioral controls (which are usually used in connection with outcome controls) are most appropriate when performance outcomes are difficult to measure due to the complex interrelationship between the environment, the task and the managerial effort. The modified Ouchi's [8] model through the use of behavior observability, which is employed in conjuction with outcome measurability and task programmability, represents an attempt to describe the factors used to decide the appropriate type of controls needed. According to that approach in cases where programmability is considered perfect the determination of output or the behaviour leads to each other with certainty and no risk is entailed. This is not the case in the systems views of an organization. When the task programmability is not perfect though then there is no strict correspondence between behaviour and outcomes, so some kind of risk is assumed as we can see in the following table (cells 5 through 8).

\begin{tabular}{|c|c|c|c|}
\hline & \multicolumn{2}{|c|}{ Task Programmability } \\
\hline & & Perfect & Imperfect \\
\hline High & High & 1 & 5 \\
\hline $\begin{array}{l}\text { Outcome } \\
\text { Observability }\end{array}$ & $\begin{array}{l}\text { behaviour } \\
\text { observability }\end{array}$ & $\begin{array}{l}\text { Output or } \\
\text { behaviour } \\
\text { control }\end{array}$ & $\begin{array}{l}\text { Behaviour } \\
\text { control }\end{array}$ \\
\hline
\end{tabular}




\begin{tabular}{l|l|c|c}
\hline & $\begin{array}{l}\text { Low } \\
\text { behaviour } \\
\text { observability }\end{array}$ & $\begin{array}{l}\text { Output } \\
\text { control }\end{array}$ & $\begin{array}{l}\text { Output } \\
\text { control }\end{array}$ \\
How & $\begin{array}{l}\text { High } \\
\text { behaviour }\end{array}$ & $\begin{array}{l}\text { Behaviour } \\
\text { Obseme } \\
\text { observability } \\
\text { control }\end{array}$ & $\begin{array}{l}\text { Behaviour } \\
\text { control }\end{array}$ \\
& $\begin{array}{l}\text { High } \\
\text { behaviour } \\
\text { observability }\end{array}$ & $\begin{array}{l}\text { Behaviour } \\
\text { control }\end{array}$ & $\begin{array}{l}\text { Behaviour } \\
\text { control }\end{array}$ \\
\hline
\end{tabular}

Fig. 1. Control Type Models

Source: [9]

\section{Performance Measurement, Strategic Controls and The Balanced Scorecard}

Strategic control is part of the process of defining strategic goals, monitoring the measurement and reward aspects of performance of private and public organizations.

This process of strategic control ensures:

\section{$>$ Effective implementation of strategy}

$>$ Measurement and comparison of actual vs. expected results in relation to predefined strategic targets

$>$ Analysis of issues and problems, developing solutions and carrying out corrective and improvement actions for the achievement of expected performance.

Performance measurement of private and public organizations is the basis for the development of a strategic control system. The standardized model of the Balanced Scorecard framework offers management a dynamic tool for the development of such a strategic control system. This system measures all the critical dimensions of organizational performance (financial, customers, internal corporate processes, and employee learning and innovation).

Strategic control entails developing performance measures that allow managers both to evaluate how well they have utilized organizational resources to create value and to sense new opportunities for creating value in the future. One increasingly influential model that guides managers through the process of creating the right kind of strategic control systems to enhance organizational performance is the balanced scorecard model. According to it, strategic managers have traditionally relied on financial measures of performance such as profit and return on investment, EVA, MVA and other, to evaluate organizational performance. Financial information, though important, is not enough by itself. If strategic managers are to obtain a true picture of organization performance, financial information must be supplemented with performance measures that indicate how well an organization has been achieving the four building blocks of competitive advantage - efficiency, quality, innovation and responsiveness to customers. This is so because financial results simply inform strategic managers about the results of decisions they have already taken; the other measures balance this picture of performance by informing managers about how accurately the organization has in place the building blocks that drive the future performance.

According to [10], strategic management system are developed in order to control a strategy implementation process by providing short-term target that can deliver long-term goal. BSC operates as a kind of constraint for the behaviour of all the agents of an organisation since their actions concerning certain dimensions are measured (directly or indirectly) regularly and the results are assessed based on predetermined targets mutually agreed upon.

The Balanced Scorecard is a tool that enables a strategy to become operational. The implementation of the BSC contributes to a successful application of the strategy, ensures the realization of the mission and goals and secures company's long-term growth and development.

The standardized model of the Balanced Scorecard framework offers management a dynamic tool for the development of such a strategic control system. This system measures all the critical dimensions of organizational performance (financial, customers, internal corporate processes, and employee learning and innovation). Although financial goals and controls are an important part of the balanced scorecard approach, they are mainly backward looking and incomplete. Thus it is also necessary to develop goals and controls that tell managers how well their strategies are creating a competitive advantage and building distinctive competences and capabilities that will lead to future success. Towards that aim Kaplan and Norton [11] developed a multi-dimensional corporate tool, that directs firm to align its performance measurement and controls from the customers' perspective, internal business processes, and learning and growth perspectives and investigate their impact on the financial indicators.

When strategic managers implement the balanced scorecard approach and establish goals and measures to evaluate efficiency, quality, innovation, and responsiveness to customers, they are using output control. Organization's reward system is usually linked to the performance of these goals, so that output control also provides an incentive structure for motivating employees at all levels in the organization

One version of the balanced scorecard operates, is presented below. It is based on an organization's mission and goals. Then an organizational structure is established to use resources to obtain a competitive advantage and achieve these goals.

Strategic control entails developing performance measures that allow managers both to evaluate how well they have utilized organizational resources to create value and to sense new opportunities for creating value in the future. One increasingly influential model that guides managers through the process of creating the right kind of strategic control systems to enhance organizational performance is the balanced scorecard model. According to the balanced scorecard model, strategic managers have traditionally relied on financial measures of performance such as profit and return on investment to evaluate organizational performance. Financial information, though important, is not enough by itself. If strategic managers are to obtain a true picture of organization performance, financial information must be supplemented with performance measures that indicate how well an organization has been achieving the four building blocks of competitive advantage - efficiency, quality, innovation and responsiveness to customers. This is so because financial results simply inform strategic managers about the results of decisions they have already taken; the other measures balance this picture of performance by informing managers about how accurately the organization has in place the building blocks that drive the future performance.

To evaluate how well the strategy and structure are working, managers develop specific performance measures 
that assess how well the four building blocks of competitive advantage are being achieved.

- Efficiency can be measured by the level of production costs, the number of hours needed to produce a product, and the cost of raw materials.

- Quality can be measured by the number of rejects, the number of defective products returned from customers, and the level of product reliability over time.

- Innovation can be measured by the number of new products introduced, the time taken to develop the next generation of new products in comparison with the competition, and the expense or cost of product development.

- Responsiveness to customers can be measured by the number of repeat of customers, the level of ontime delivery to customers, and the level of customer service.

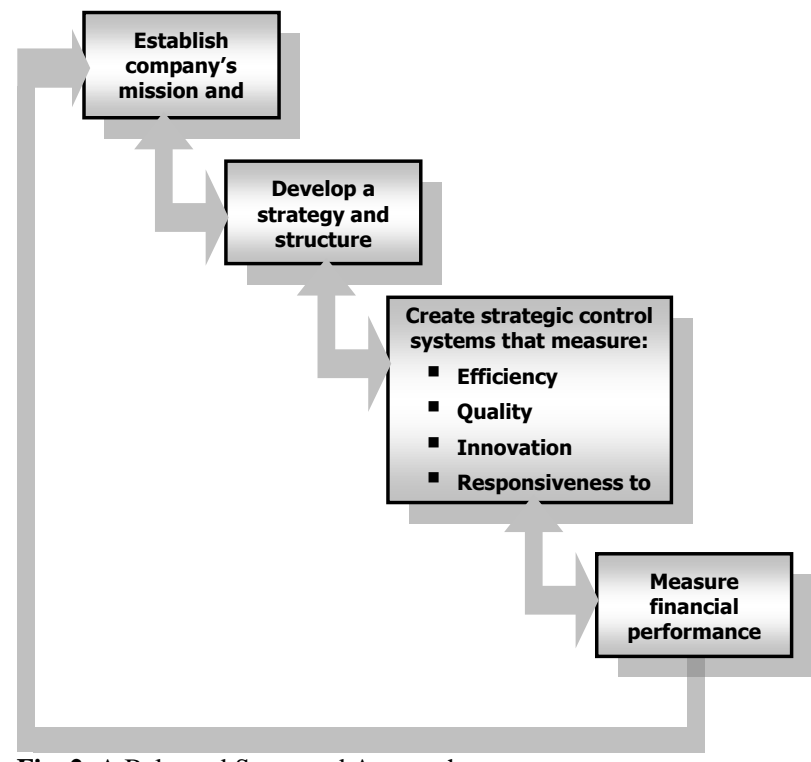

Fig. 2. A Balanced Scorecard Approach

BSC operates as a kind of constraint for the behaviour of all the agents of an organisation since their actions (concerning certain critical dimensions) are measured (directly or indirectly) regularly and the results are assessed based on predetermined targets mutually agreed upon.

A typical BSC is the following one:

FINANCIAL AREA CONTROLS

\begin{tabular}{|c|c|}
\hline Strategic Objectives & Strategic Measures \\
\hline Financial performance & $\begin{array}{l}\text { liquidity, cash flows, } \\
\text { profits, return on sales, } \\
\text { equity capital employed, } \\
\text { return on assets and equity, } \\
\text { EVA, MVA, ROI, ROCE, } \\
\text { RNOA } \\
\text { Stock Price, Cash Flow }\end{array}$ \\
\hline \multicolumn{2}{|c|}{ CUSTOMER AREA CONTROLS } \\
\hline Strategic Objectives & Strategic Measures \\
\hline $\begin{array}{ll}\text { Increase } & \text { Customer } \\
\text { Satisfaction } & \end{array}$ & $\begin{array}{l}\text { Market share } \\
\text { Customer Retention Ratio } \\
\text { Customer Satisfaction } \\
\text { Survey }\end{array}$ \\
\hline
\end{tabular}

\begin{tabular}{|l|l|}
\hline INTERNAL PROCESS AREA CONTROLS \\
\hline Strategic Objectives & $\begin{array}{l}\text { Strategic Controls } \\
\text { (Measures) }\end{array}$ \\
\hline $\begin{array}{l}\text { Innovative Products/Services } \\
\text { Creation }\end{array}$ & $\begin{array}{l}\text { No of Patents of sales due to new } \\
\text { products or services, time of } \\
\text { response, } \\
\text { \% of defect products or } \\
\text { complaints per } \\
\text { Number of accidents per } \\
\text { year new } \\
\text { Acceptance of } \\
\text { products/services } \\
\text { New products/service, Cycle } \\
\text { time }\end{array}$ \\
\hline Improve \\
understanding
\end{tabular}

\section{Performance Measurement in a turbulent Environment}

Tavakoli and Perk [12] pointed out that as the environment is changing constantly, strategic management must have the ability to take into account both deliberate and emergent strategies. Performance measurement models, it is true that they are largely based on deterministic assumptions about the world. The adoption of uncertainty, bounded instability, and self-organization in the business world, is changing the context of application. Palmer and Parker [13] conclude that performance systems must be realigned with knowledge on uncertainty and thus should focus on identification of the "aggregate system".

The traditional BSC framework must adjusted accordingly to accommodate a turbulent external environment. It must be agile and flexible. That is why some researchers hold that "A key dimension of strategic navigation is fast forward creativity, which reflects a flexible and informal but targeted control focus More conventional ideas of control, including balanced scorecards and interactive control may lack flexibility and be too restrictive in helping organizations to adapt effectively and rapidly to varying and uncertain external circumstances" [14]. Some others also feel that "although a state of balance in the performance measurement system is a theoretically appealing ideal "small, rapidly growing firms in turbulent conditions often may find themselves in a state of 
imbalance. Given the nature of the conditions under which such firms operate, some degree of imbalance may even be a useful feature of their management control package. Small firms are vulnerable and dominated by an informal logic. When they grow rapidly, they face numerous tensions. If this occurs in a turbulent environment, changes are frequent, profound and unpredictable. It is necessary for such firms to quickly detect and respond Balancing the management control package" [15]. Other authors though establish an integrated model of Balance Scorecard and Objective Matrix, point out that "the flexibility and easiness to maintain the performance is very important for the top manager in rapid changing business environment. The feel that the performance management focused on customers views is important, since the satisfied customer will increase the loyalty which contributes to the organization revenues" [16]..

Increased volatility of the business environment makes systematic strategic planning more difficult. Rapid change requires strategies that are flexible and creative according to Grant [17]. He explains that "Strategic planning processes have become more decentralized, and more informal, while strategic plans themselves have to become of shorter term, more goal focused, and less specific with regard to actions and resource allocations. Strategic planning has to become less about strategic decision making and more a mechanism for coordination and performance managing. Managers faced with a constantly changing fitness landscape, maximizing survival (reaching high fitness peaks) implies constant and parallel exploration.

\section{Conclusions}

The performance evaluation culture that is transplanted in to the organizations adopting the Balanced Scorecard apparatus, is operating as a binding mechanism that canalizes the transformation process of it should the environment changes. Given that all components of the organization are measured regularly against specified targets emanating from the agreed strategy, it minimizes or even prevents non desirable behavior, actions and outcomes which affect adversely the remuneration package of all agents involved. It finally constraints the transformation process and operate as a strange attractor which explore the business fitness landscape directing organization towards an agreed road map contributing to its survival and further development.

\section{References}

1. Teece, D. J., Gary, P., Amy, S., 1997. "Dynamic capabilities and strategic management". Strategic Management Journal 18 (7), 509-533.

2. Reigeluth C. M (1996) "Chaos Theory and the Sciences of Complexity: Foundations for Transforming Education".

3. Ferdig M. "Complexity Theories: Perspectives for the Social Construction of Organizational Transformation "

4. Eisenhardt K. M. (1985). "Control: Organizational and Economic Approaches”. Management Science, Vol. 31, No 2, Feb, pp 134-149.

5. Curtis P., Politou D., and Kyriazoglou J. ( 2007). “ Strategic Controls and the Balanced Scorecard framework.

6. Neely, A. (2000). Editorial: Performance measurement: theory and practice. International Journal of Performance Management, 2(1/2/3), 1

7. Kueng, P., Meier, A., \& Wettstein, T. (2001). Performance Measurement must be Engineered. Communications of the Association of Information Systems, 7(3).

8. Ouchi, W. F. (1980). "Markets, Bureaucracies, and Clans", Administrative Science Quarterly, Vol. 25, pp $129-141$

9. Govindarajan V. and Fisher J.(1990). "Strategy, Control Systems and Resource Sharing :Effects on Business-Unit Performance". Academy of Management Journal. Vol. 33, No. 2, pp 259-285

10. Atkinson, H. (2006): "Strategy Implementation: A Role for the Balanced Scorecard?" Management Decision, Vol. 44 (10), pp. 1441-1460.
11. Kaplan R. S. and. Norton D. P.(1996). "Using the Balanced Scorecard as a Strategic Management System", Harvard Business Review, January-February 1996, pp 75-78)

12. Tavakoli, I. and Perk, K., (2001). "The development of a strategic control sy tem for the management of strategic change. Strategic Change 10, 297-305.

13. Palmer E and Parker D.(2001) "Understanding performance measurement systems using physical science uncertainty Principles". International Journal of Operations \&

Production Management, Vol. 21 No. 7, pp. 981-999.

14. Durden C., and Perera H. (2010). " Towards control using strategic navigation in turbulent organisational environments" International Journal of Accounting, Auditing and Performance Evaluation, Volume 6, Number 4, p.p 397 - 421

15. Nilsson A. (2010). " Balancing the management control package of small, rapidly growing firms in turbulent environments". Int. J. Entrepreneurship and Small Business, Vol. 11, No. 1,

16. Sulisworo, D and Darmawati D, (2011). Balance Scorecard and Objective Matrix Integration for Performance Targeting Method of Infocom Business. "Indian Journal of Commerce \& Management Studies Vol-II , Issue -4 May 2 pp 50-60

17. Grant R. M. (2003) "Strategic Planning in a Turbulent Environment: Evidence from the Oil Majors. Strategie Management Journal, Vol.24, :491-517 